\title{
L'Institut international des films du travail, 1953-1972. Formation syndicale et propagande par le cinéma dans le contexte de la guerre froide
}

The International Labour Film Institute (1953-1972). Workers' Education and Propaganda through Film in the Context of the Cold War

\section{Françoise F. Laot}

\section{OpenEdition Journals}

Édition électronique

URL : https://journals.openedition.org/itti/1234

DOI : $10.4000 /$ itti. 1234

Éditeur

Université de Poitiers

Référence électronique

Françoise F. Laot, « L'Institut international des films du travail, 1953-1972. Formation syndicale et propagande par le cinéma dans le contexte de la guerre froide », Images du travail, travail des images [En ligne], 2 | 2016, mis en ligne le 01 septembre 2016, consulté le 26 juillet 2021. URL : http:// journals.openedition.org/itti/1234; DOI : https://doi.org/10.4000/itti.1234

Ce document a été généré automatiquement le 26 juillet 2021.

Images du travail, travail des images 


\section{L'Institut international des films du travail, 1953-1972. Formation syndicale et propagande par le cinéma dans le contexte de la guerre froide}

The International Labour Film Institute (1953-1972). Workers' Education and Propaganda through Film in the Context of the Cold War

\section{Françoise F. Laot}

1 Cet article entend contribuer à éclairer la relation qu'ont entretenue cinéma et syndicalisme dans l'histoire. Il se centre sur l'activité d'un institut international, l'ILFI (International Labour Film Institute) issu du mouvement syndical mondial, qui a consacré son activité à l'éducation ouvrière par le cinéma, notamment, en organisant cinq festivals en différents endroits du monde. Après avoir exposé les raisons de la création de cet institut, nous suivrons les évolutions de son action au fil du temps. Nous montrerons que si elles tiennent en partie à des raisons internes à l'institut, elles doivent aussi beaucoup aux grands changements géopolitiques et technologiques qui se sont produits sur la période étudiée. Nous reviendrons également sur quelques débats qui ont animé l'ILFI, sur ce qu'est un « bon » film syndical, sur ce qu'il doit montrer, sur ceux et celles qu'il a oublié-e-s de mettre à l'écran. Enfin nous verrons que l'avènement de « l'audiovisuel » dans la formation a profondément modifié la donne en détrônant le cinéma et comment cette évolution a progressivement conduit à la dissolution de l'ILFI en 1972.

2 Cette recherche s'appuie essentiellement sur les archives de l'ILFI et de la Confédération internationale des syndicats libres (CISL) qui se trouvent à l'Institut international d'histoire sociale à Amsterdam (IISH) ${ }^{1}$, ainsi que sur des comptes rendus des congrès des organisations syndicales internationales (OSI) et sur de nombreux 
articles parus dans leurs organes de presse, pour la plupart consultés à l'Institut Souvarine de Nanterre et dans les centres d'archives des centrales françaises².

Avant d'entrer dans le vif du sujet, il nous faut souligner la polysémie du terme anglais «labour ${ }^{3}$ ", signifiant «travail » mais aussi « les travailleurs » ou « le monde ouvrier ", comme dans l'expression labour movement (mouvement ouvrier). La traduction française, «films du travail », qui a finalement été retenue par ses fondateurs euxmêmes dans l'intitulé de l'ILFI, ne rend pas compte de ces deux dimensions enchevêtrées. Celles-ci expliquent pourtant en grande partie certains des choix et les ambiguïtés apparentes de l'institut.

\title{
1. Le film : une arme syndicale à l'échelle internationale
}

\author{
«[...] une partie considérable du mouvement \\ ouvrier du monde libre a, jusqu'à l'heure actuelle, \\ totalement méconnu l'importance capitale du \\ film, en tant que moyen de propagande et \\ d'éducation populaire. Pourtant, nos adversaires, \\ en particulier les maîtres des régimes totalitaires, \\ de quelque couleur qu'ils soient, ont, depuis de \\ longues années, tiré un profit très large des \\ possibilités immenses qu'offre le cinéma [...] \\ Même s'il est hostile aux idées qu'on veut lui faire \\ accepter, le public ne reste pas insensible aux \\ arguments mensongers mis en image. [...] \\ N'hésitons donc pas à dire que, du point de vue \\ propagande et du point de vue éducation, il n'y a \\ pas d'arme plus puissante que le cinéma ${ }^{4}$.»
}

\subsection{Cinéma et mouvement ouvrier : une histoire conflictuelle}

Le mouvement ouvrier ne prend que lentement conscience de l'importance du cinéma pour l'action syndicale (Perron, 1995, 2000 ; Tartakowski, 1996). En 1995, Tangui Perron lançait un appel pour qu'histoire sociale et histoire du cinéma se croisent enfin (Perron, 1995, 23). De fait, des travaux de la sorte ont été publiés au cours de ces dernières années. Quelques recherches se sont récemment intéressées au cinéma militant dans les années 1968 ou encore à la représentation des ouvriers et des ouvrières dans les films documentaires ou de fiction (Hatzfeld et al., 2009 ; Hatzfeld, 2013 ; Rochet et al., 2015). Cependant, les approches internationales, comme celles que Marcel van der Linden (2012) voudrait voir se développer sur l'histoire du mouvement ouvrier, restent rares.

En France, si la CGT et le cinéma naissent la même année comme le met en valeur le numéro du Mouvement social dirigé par Pierre Sorlin, célébrant ce double centenaire en 1995, la confédération s'est longtemps montrée très méfiante voire méprisante à l'égard de ce qu'elle considérait comme une attraction vulgaire et amorale. Le cinéma n'est-il pas également l'expression patronale d'un «capitalisme conquérant " comme peuvent le suggérer les premières images de la sortie des usines Lumière (Perron, 1995, 
21) ? À contre-courant pourtant, se crée en 1913 la Coopérative ouvrière : le Cinéma du peuple, qui invente le cinéma agit-prop et qui réunit quelques syndicalistes et de nombreux anarchistes, eux aussi pourtant d'abord réticents vis-à-vis du cinéma qui avait servi aux forces de l'ordre à identifier les émeutiers lors de grèves (Jarry, 2001). Cette expérience amorce un changement radical dans les représentations du cinéma chez certains syndicalistes qui le voient dorénavant comme un puissant moyen d'éducation et de contre-culture. Le mouvement libertaire confirme par la suite son attachement au cinéma ${ }^{5}$, notamment à travers l'action de diffusion des films des maisons du peuple en Belgique où elles sont bien implantées. Cette forme de cinéma marginale côtoiera, sans le rencontrer, le " cinéma éducateur " promu par la Ligue de l'enseignement, considéré comme acceptable pour le mouvement social, notamment au sein de la SFIO, pour les services qu'il rend en matière de propagande hygiéniste et antialcoolique et qui connaît son âge d'or dans les années 1920 (Borde, Perrin, 1992 ; Vignaux, 2007; Laborderie, 2015). Pour le mouvement ouvrier, dans l'entre-deuxguerres, c'est aussi la découverte du cinéma soviétique que le Parti communiste entreprend de diffuser. Dans les années 1930, d'autres groupes militants de production de cinéma (parfois aux côtés du théâtre ou d'autres arts) réunissant syndicalistes, anarchistes, communistes et gens du spectacle sous un mode coopératif, verront le jour comme le groupe Octobre (1932) ou, sous le Front populaire, Ciné-Liberté (Ory, 1975).

6 Une première initiative internationale, mais non syndicale, autour du cinéma voit le jour au sein de la Société des Nations en 1929, année de la création de l'Institut international du cinématographe éducatif. Cet institut est porté par La Coopération intellectuelle, ancêtre de l'Unesco. Mais en Italie, les partisans de Mussolini s'appuient sur le financement de cet institut pour réaliser des films au service du fascisme (Tallibert, 1998). La Seconde Guerre mondiale confirme à grande échelle le cinéma en tant qu'outil de propagande totalitaire. Au début des années 1950, cette histoire récente est évidemment encore très présente et pesante. C'est en grande partie pour contrer ces dérives "hitléro-fascistes" comme l'écrit Goerg Reuter, syndicaliste allemand leader du $\mathrm{DGTB}^{6}$, que des syndicalistes décident d'éditer leurs propres films et de créer l'ILFI'. 


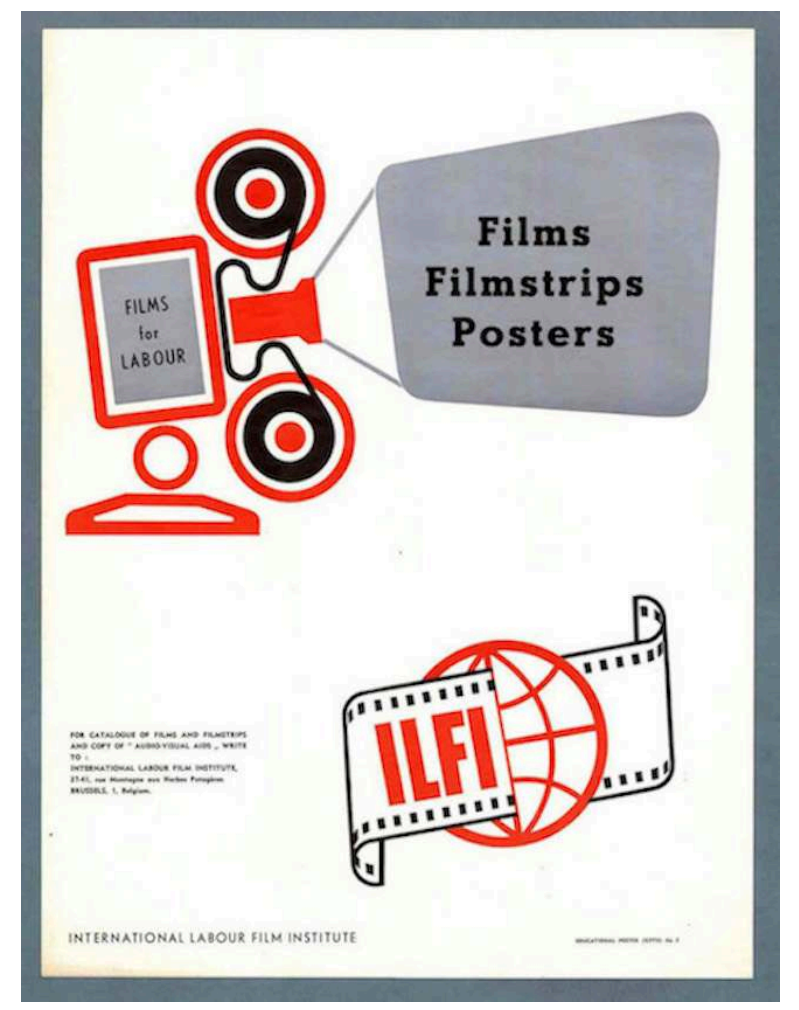

\subsection{L'ILFI, le bras armé de la Confédération internationale des syndicats libres}

7 L'entrée dans la guerre froide a provoqué, en 1949, une scission au sein de la Fédération syndicale mondiale (FSM) et donné naissance à la Confédération internationale des syndicats libres (CISL). Celle-ci, dominée par les syndicats du nord de l'Europe et de l'Amérique $^{8}$, porte son engagement sur un plan politique autant que syndical. Elle défend la démocratie contre tous les totalitarismes et se pose comme clairement anticommuniste, même si, sur ce dernier point, la position des états-uniens et des européens évoluera de manière sensiblement différente (Carew, 2000). Pour la CISL, comme le soulignent fortement ses statuts, l'éducation des travailleurs est une priorité. Or, le film est réputé être le moyen le plus sûr de toucher les masses. La confédération se lance, dès sa création, dans la confection de courts métrages et de films fixes - un support aujourd'hui oublié qui a beaucoup servi en formation ${ }^{9}$ (Goutanier et Lepage, 2008) - et met en place une première coordination internationale d'information et de diffusion des films produits par les syndicats. La décision de créer une institution spécifique chargée de développer cette activité autour du film se décide en 1952 à New York où s'est réunie sa Commission exécutive.

8 L'ILFI est officiellement créé en mai 1953 par des membres de la CISL qui se sont associés à des représentants de la Fédération internationale des associations des Maisons du peuple (FIAMP) dont le siège est alors en Suède. Ce double patronage s'avèrera tout relatif car la FIAMP est loin de peser le même poids que la CISL. Le choix institutionnel se porte sur une association de droit privé belge, indépendante 
juridiquement, mais néanmoins étroitement liée à la CISL. Les bureaux de l'ILFI sont installés près du siège de la Confédération à Bruxelles.

L'importance que les responsables de la CISL accordent à cette création se lit à travers l'implication de ses propres leaders dans les instances de l'ILFI. En effet, les membres fondateurs sont, pour la CISL, le Hollandais, Jacobus Hendrik Oldenbroek, Secrétaire général de la CISL de 1949 à 1960, les Belges, Louis Major, Secrétaire général de la Fédération générale du travail de Belgique (FGTB) et Walter Schevenels, qui avait été secrétaire de la Fédération syndicale internationale (1930-1945) et un temps pressenti comme Secrétaire général de la CISL ${ }^{10}$. La FIAMP, quant à elle, est représentée par son Président, le Suédois Karl Kilbom et par le Belge Louis Leclerc. La présence importante de membres belges dans les instances est une conséquence des conditions de la loi belge sur les ASBL (associations sans but lucratif), dont les fondateurs doivent être majoritairement des ressortissants de ce pays. Cette composition ne reflète donc pas nécessairement une implication réelle dans l'action de l'ILFI. D'autres membres éminents de la CISL vont y jouer un rôle de premier plan. En particulier, la mission éducative des films est affirmée par les liens très forts entre l'ILFI et le département Éducation de la CISL, comme le montre la succession des secrétaires du Conseil de l'ILFI, qui sont le plus souvent également responsables de ce département. C'est le cas d'Hans Gottfurcht ${ }^{11}$ (1953-1959), d'Herbert Tulatz ${ }^{12}$ (1964-1968) ou encore de Daniel Pedersen ${ }^{13}$ (1970-1972).

10 Oldenbroek restera président de l'ILFI jusqu'à la mi-1959, où il démissionnera « estimant que l'intérêt qu'il éprouve pour le cinéma et ses conceptions en la matière ne justifient pas suffisamment qu'il continue [son mandat] ${ }^{14}$. Cette démission coïncide avec deux crises, l'une à la tête de la CISL, d'où Oldenbreok est débarqué par les étatsuniens (Windmuller, 1961; Carew, 2000), l'autre interne à l'ILFI comme nous le verrons plus loin. Louis Major deviendra alors président par intérim. Il sera par la suite confirmé dans la fonction et restera président 11 ans. L'adhésion à l'ILFI est ouverte à des organisations nationales et internationales. Dans les faits toutefois, à part quelques rares exceptions ${ }^{15}$, ses membres sont les principaux syndicats membres de la CISL.

11 Les statuts de l'ILFI publiés au Moniteur belge, en août 1953, en précisent l'objet : il s'agit de promouvoir des «films consacrés au travail», expression qui comprend « des films documentaires, éducatifs et d'apprentissage, ainsi que des films récréatifs tendant à renforcer les institutions démocratiques ${ }^{16}$. $\gg$

Cette définition extrêmement floue des «films du travail», qui ne précise pas davantage qui doit les financer ou les réaliser, ne sera jamais vraiment précisée, même si, régulièrement, les archives font état de discussions sur le périmètre des films à prendre en considération.

\subsection{Les premiers pas de l'ILFI et la création d'un festival}

Pour lancer l'action de l'ILFI, ses administrateurs ${ }^{17}$ recrutent Rudolf Berner, un journaliste anarchiste suédois. Sa première tâche consiste à établir une liste de « films ouvriers » déjà existants, afin de les faire largement connaître et d'organiser leur prêt. Comme de nombreux films syndicaux sont déjà disponibles, les responsables décident de créer un «festival de films ouvriers " (ce n'est qu'à partir du deuxième festival que la dénomination sera celle de "films du travail »). La première rencontre a donc lieu à Hambourg du 15 au 18 septembre 1954. Notons qu'au même endroit, juste avant le 
festival, a lieu le congrès de la FIAMP, ce qui laisse penser que cette fédération a tout de même été influente, du moins au début de l'ILFI. À ce premier rendez-vous, participent des représentants de 12 pays «européens et extra-européens ", ainsi que des délégués des Syndicats professionnels internationaux.

Illustration 2. Couverture des actes du premier festival de l'ILFI à Hambourg (1954) avec l'affiche d'un film japonais.

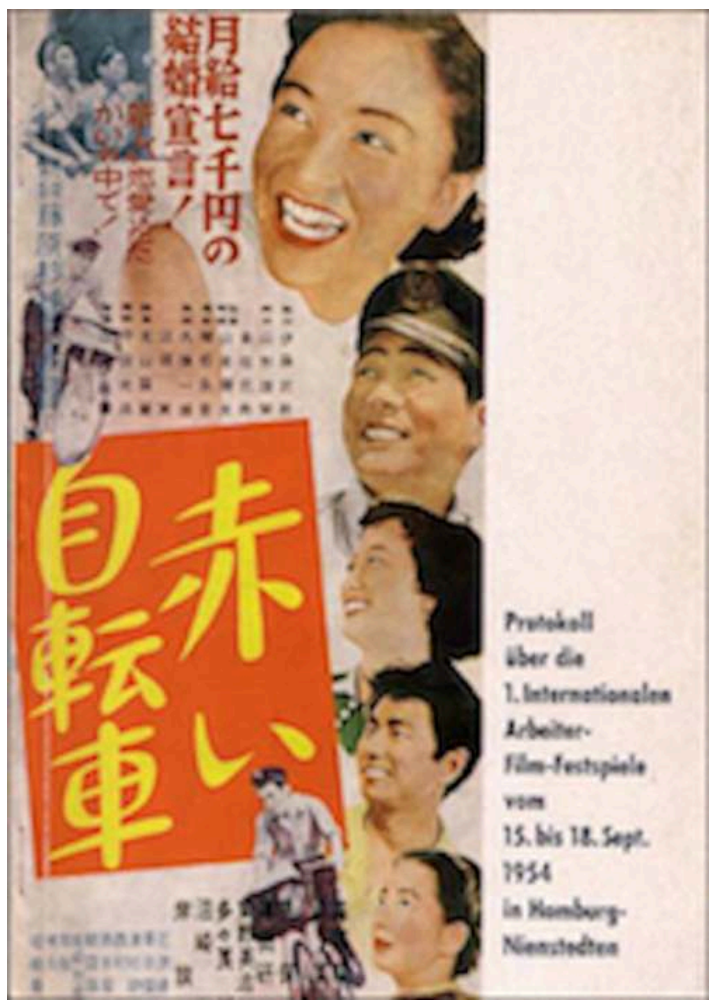

Le 15 septembre, à la cérémonie d'inauguration du festival, se succèdent les discours de Georg Reuter, d'Hans Gottfurcht, sur laquelle nous reviendrons, et de Karl Kilbom.

Une sélection de 26 films concourant sous 4 catégories différentes (voir tableau infra) y est présentée. Les projections de films alternent avec des conférences et des débats. La haute distinction du jury est décernée au film suédois När ängarna blommar (Quand les champs sont en fleurs) (1947) de Jan Friedegard et Gösta Netzen. Il s'agit d'une fiction produite en collaboration avec le syndicat suédois des travailleurs de la terre, qui raconte l'histoire d'un conflit entre un fermier et ses ouvriers agricoles et du meurtre d'un briseur de grève.

16 À l'issue du festival, les participants prennent une résolution: faire un nouveau festival, dans un délai de deux ans «au plus tard» et former un groupe de travail d'experts de films ouvriers réunissant des syndicalistes et des techniciens du cinéma sympathisants. L'ILFI se donne pour mission de rechercher l'adhésion des syndicats et fédérations nationales, du mouvement coopératif international, des associations de tourisme ouvrier et des centres éducatifs ouvriers, en qualité de membres actifs et cotisants... Il est demandé aux organisations membres de fournir une copie gratuite des films qu'elles produisent à l'ILFI qui a également un rôle de conseil : il peut donner un avis sur « l'intérêt international » des scénarios qu'on lui soumet. 
«Il est recommandé aux fédérations syndicales nationales de réaliser des films instructifs, en collaboration avec les ministères du travail de leurs pays respectifs, afin d'encourager la connaissance des problèmes éducatifs et professionnels ${ }^{18}$. 》 dans une forme guerrière la devise de la CISL :

«Le film est une arme puissante dans la lutte pour le PAIN, la PAIX, et la

LIBERTÉ ${ }^{25}$. »

Il leur est également demandé d'intervenir auprès des gouvernements de leurs pays pour que soit ratifiée la convention de l'Unesco pour un échange international des films éducatifs sans barrière douanière. L'ILFI sera par la suite membre du Conseil international du cinéma et de la télévision créé en $1958^{19}$.

L'institut enfin a aussi pour mission de prêter du matériel cinématographique et des équipements audiovisuels aux syndicats membres qui en font la demande.

\subsection{Répertorier les films du travail déjà existants}

Le catalogue des films du travail paraît en juin 1956 en cinq langues (français, anglais, allemand, italien et suédois). Il est précisé en introduction :

« Non seulement les films réalisés par, ou pour, les organisations ouvrières, mais également les films d'autres provenances devaient trouver leur place dans cet aperçu pour autant qu'ils fussent d'un emploi utile pour la CISL, ses organisations affiliées, en particulier en vue de leurs programmes de formation et d'éducation, et pour les autres organisations membres de l'ILFI [...]. Il fut entendu que les films documentaires et les films s'occupant de problèmes syndicaux constitueraient la majorité des films catalogués, mais des films divertissants présentant un caractère social ne devaient pas être exclus ${ }^{20}$. »

Le catalogue présente 345 films et 66 films fixes classés par pays. Les titres sont ensuite repris dans un classement par catégories ${ }^{21}$, puis par ordre alphabétique. Il ne s'agit pas à proprement parler "d'agit-prop ». Pour la très grande majorité, ce sont des films tournés en $16 \mathrm{~mm}$ et produits dans la décennie précédente. La plupart sont des courts métrages d'une durée moyenne de 15 à $20 \mathrm{~min}$. Pour la France, un seul film figure : Jour de peine (1952) de Victor Vicas qui raconte la lutte ouvrière dans les aciéries du Nord. Ce faible nombre, comparé par exemple aux 14 films autrichien, aux 23 danois ou aux 47 suédois... s'explique par le fait que le syndicat membre français de l'ILFI, comme celui de la CISL, est la CGT-FO, syndicat encore très jeune en 1956, contrairement aux syndicats d'autres pays ${ }^{22}$. À l'exception de Rose Étienne ${ }^{23}$, qui participera à quelques réunions de l'ILFI avant de prendre sa retraite en 1963 - sans toutefois faire partie des instances décisionnelles ${ }^{24}$-, les responsables de FO n'y sont pas présents.

Le cinéma est investi d'un pouvoir fort par les rédacteurs du catalogue qui reprennent

Quatre autres festivals seront organisés après celui de Hambourg (cf. tableau infra) : Vienne, 1957, Stockholm, 1960, Tel-Aviv, 1963 et Montréal, 1967. 


\section{Des films du Nord pour le Sud dans la compétition entre l'Est et l'Ouest : une stratégie hasardeuse}

\section{1. Éducation et propagande : les « bons films » à montrer en période postcoloniale}

Les films servent-ils l'éducation ou bien la propagande? La frontière entre ces deux options est ténue sous la plume de nombreux syndicalistes impliqués dans l'ILFI tout comme à la CISL qui, selon ses statuts, se doit «d'entreprendre et d'encourager une œuvre d'éducation et de propagande dans le but d'augmenter les connaissances et le degré de compréhension des travailleurs à l'égard des problèmes nationaux et internationaux qui se posent à eux, leur permettant ainsi de lutter de manière plus efficace ${ }^{26}$. " L'éducation syndicale, qui constitue le coeur de l'action de l'action de la confédération internationale, a une visée émancipatrice. Ainsi qu'il est proclamé à son Congrès mondial en 1953 :

«Le but ne sera atteint que lorsque la masse des travailleurs et ceux qui les représentent auront acquis le savoir et l'expérience nécessaires pour changer les structures de la société afin d'éradiquer à tout jamais la peur et la misère ${ }^{27}$. "

Tandis que le film documentaire informe et transmet des connaissances au monde des travailleurs, «le bon film de propagande doit montrer les conquêtes du mouvement ouvrier ${ }^{28}$.»

La conférence de Gottfurcht au Festival de Hambourg, intitulée "The Labour Movement and the Film"29 lui donne l'occasion de revenir sur la valeur éducative du cinéma.

Il précise :

« Tout comme il existe une bonne et une mauvaise éducation, il y a des bons et des mauvais films [...] les films peuvent contribuer à l'éducation ou à l'abrutissement (stultification), ils peuvent encourager une saine démocratie ou entretenir l'indifférence politique. Ils peuvent critiquer les conditions sociales, lorsqu'elles méritent de l'être et encourager les progrès. Ils peuvent instruire comme divertir, amuser ou stimuler, réjouir ou terrifier... Dans notre champ d'action, les films sont une arme dans l'arsenal éducatif du mouvement ouvrier et il est un important facteur d'éducation et de formation ${ }^{30}$.

Il continue en détaillant le rôle que devraient jouer les syndicats, par exemple en influençant la production de films ou en produisant les leurs, car souligne-t-il :

« il n'y a pas de propagande bon marché. La bonne propagande a un prix, mais la

mauvaise coûte également beaucoup d'argent ${ }^{31}$. »

Gottfurcht ne méprise pas les fictions. Selon lui elles peuvent permettre à des travailleurs fatigués de se tourner vers des loisirs sensés et les conduire à s'intéresser à des sujets valables. Comme les autres arts, les films de fiction peuvent amener le grand public à prendre en compte les intérêts du mouvement ouvrier dont une des tâches consiste à préparer les esprits pour une participation progressive de tous à la vie sociale.

En 1958, il défendra Quelqu'un frappe à la porte, cette fiction réalisée par Alexandre Szombati ${ }^{32}$ recruté en tant que directeur de l'ILFI en 1957. Le film raconte une grève sur fond d'idylle entre un travailleur hongrois qui a fui son pays et la petite fille du « vieux syndicaliste » qui l'accueille au sein de sa famille à Gant. C'est un mixte de la biographie de son réalisateur et une charge contre les communistes, ces derniers étant ceux qui - 
heureusement sans succès - se mettent en travers de l'action syndicale. Le film sera le seul long métrage produit par l'ILFI. Il provoque une grave crise qui tient d'ailleurs beaucoup moins à son contenu qu'à son coût exorbitant qui conduit l'ILFI au bord de la ruine. J'ai décrit ailleurs les débats internes et les conséquences institutionnelles auxquels il a donné lieu (Laot, 2015a). Retenons seulement ici qu'il entraîne le départ de Szombati en mai 1961 et une réorganisation complète de l'ILFI avec son recentrage sur une activité de prêts et de doublage de films, notamment pour une diffusion dans les pays en voie de développement.

Photographie 1. Image du film Quelqu'un frappe à la porte (Szombati, ILFI, 1958).

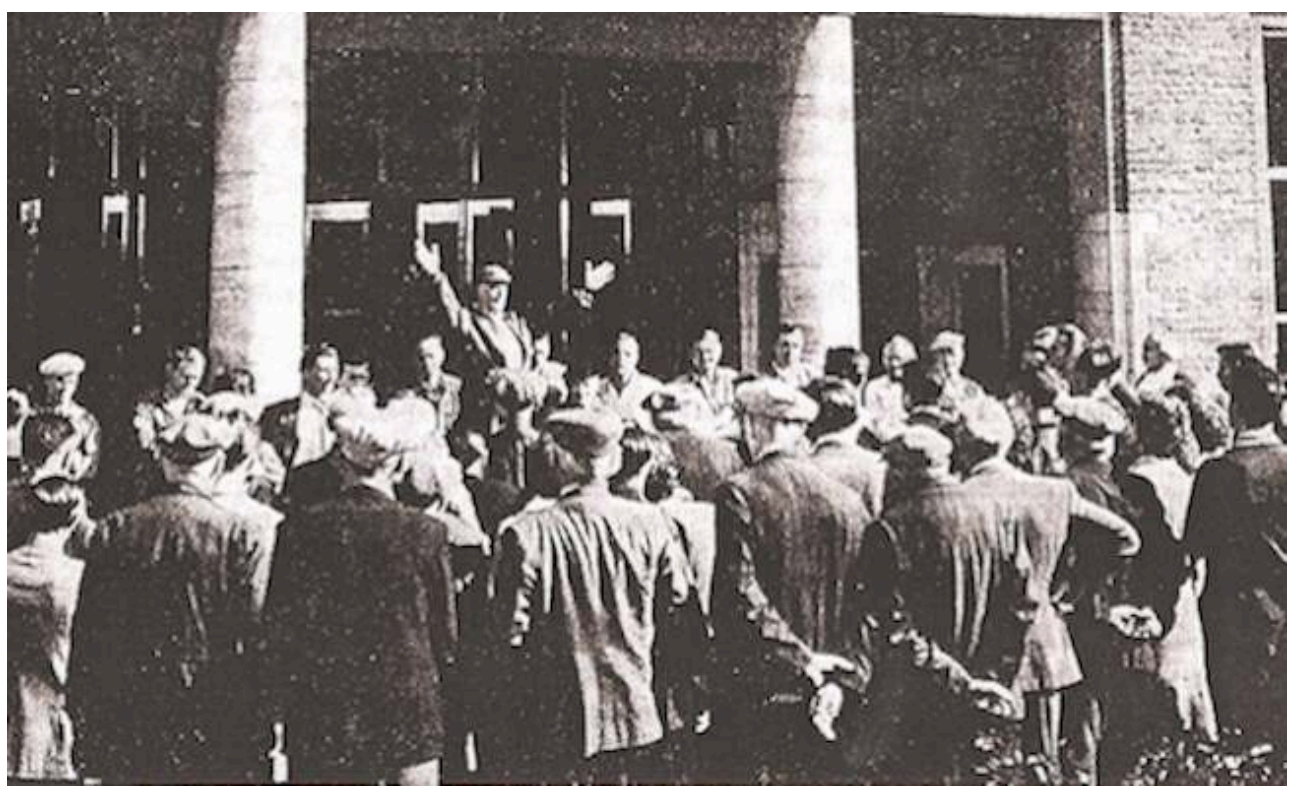

Les conférences données au festival de Stockholm sont d'autres occasions de revenir sur la valeur éducatrice du cinéma. Dans une approche très humaniste, Joseph Burstein, israélien ${ }^{33}$, membre du comité consultatif de l'ILFI, y déclare :

«Les films ne sont pas faits pour donner des solutions [... leur] tâche est accomplie quand [ils] montrent le besoin et, par-dessus tout, le désir d'arriver à une solution. [...] C'est le meilleur moyen d'apprendre aux millions de spectateurs ce qui a uni les différentes races et peuples et ce qui conduit à s'élever contre la répression ${ }^{34}$.»

31 Selon lui, il serait bon de montrer l'indépendance des pays d'Asie et d'Afrique et l'épanouissement de leurs peuples "sous l'œil sensible de la caméra " ainsi, "les travailleurs européens et américains, en les voyant, auraient leur humanité naturelle réveillée et rafraîchie ${ }^{35}$.»

Pourtant, dans le cadre de la décolonisation des années 1960, la CISL connaît quelques déboires. Sa volonté hégémonique et ses positions souvent atlantistes, l'amènent à commettre des «maladresses psychologiques » (Espéret, 1965, 454), notamment auprès des jeunes syndicats $d u$ « tiers-monde». 


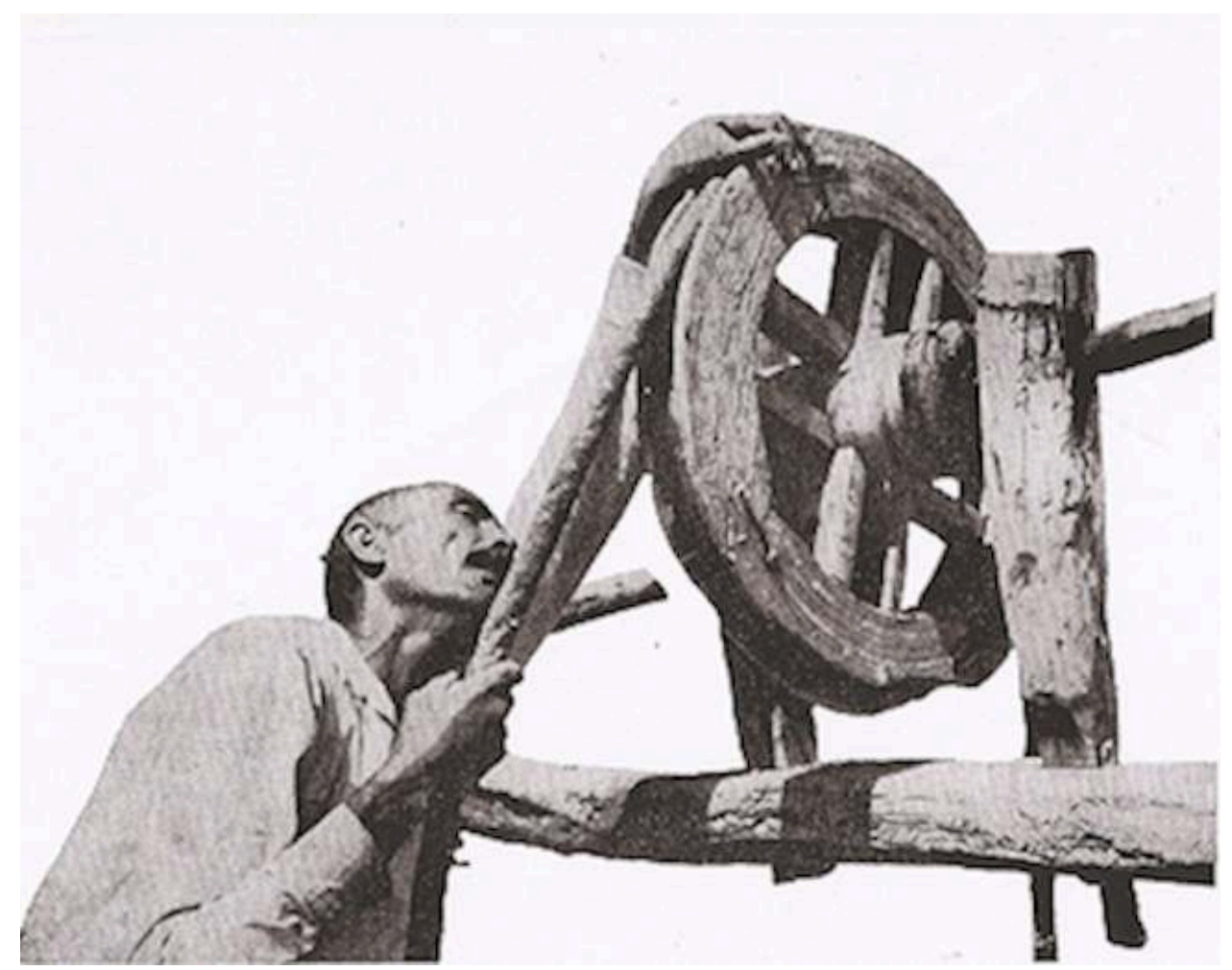

L'Afrique, en particulier, est un terrain privilégié pour le développement d'actions éducatives de toutes sortes en direction des syndicalistes, menées par les organisations syndicales internationales ainsi que par les syndicats états-uniens. Selon Marcel van der Linden (2000: 533), ce besoin d'éduquer relève d'une vision qui reste enracinée dans l'idéologie coloniale. La CISL, considérant que certains peuples ne sont pas prêts à prendre leur destinée en main, souhaite imposer comme modèle les syndicats des pays industrialisés. Les pays en développement sont ainsi le théâtre d'une rude compétition entre les deux grandes organisations syndicales internationales, la FSM et la CISL. Il s'agit, par l'adhésion de leurs syndicats, de faire pencher les pays émergents du «bon côté ». À ce jeu-là cependant, la CISL est mise en difficulté, souvent accusée par les syndicalistes des jeunes nations de faire passer l'anticommunisme avant l'anticolonialisme (Carew, 2000)... C'est ainsi qu'elle en vient à perdre du terrain face à la FSM, sa grande rivale.

On le voit, ces enjeux de communication dépassent de beaucoup la question de la formation syndicale.

Pour Walter Reuther, le leader du puissant United Automobile Workers of America (UAW) et vice-président de la CISL, qui a rejoint le conseil d'administration de l'ILFI, montrer des films fait littéralement partie de l'arsenal de la guerre froide. Il estime en effet qu'ils peuvent «corriger l'image déformée des syndicats de l'Ouest qui a été soigneusement diffusée par les communistes en Afrique, en Asie et en Amérique latine ${ }^{36}$ " et il propose qu'un comité choisisse les films "les plus adéquats » afin d'en faire des doublages dans le plus grand nombre de langues possibles. Il est prêt à mettre sur la table des sommes conséquentes, à hauteur de 25000 dollars par an, pour que cette politique de synchronisation soit développée. En 1962, l'ILFI, qui se remet très difficilement de son grave déficit, saisit cette opportunité pour se remettre à flot et 
envisager sereinement l'organisation d'un prochain festival. L'institut restera sous perfusion financière de l'UAW jusqu'à la fin des années 1960.

\subsection{Une internationalisation inachevée}

Tableau : Les festivals de l'ILFI.

\begin{tabular}{|c|c|c|c|c|}
\hline 15-18 septembre 1954 & $2-6$ juin 1957 & $22-27$ mai 1960 & 5 au 10 mai 1963 & 11-15 août 1967 \\
\hline Hambourg & Vienne & Stockholm & Tel-Aviv & Montréal ${ }^{*}$ \\
\hline $\begin{array}{c}\text { Participants : } \\
\text { des représentants de } 12 \text { pays } \\
\text { europénss et « extra-européens » }\end{array}$ & $\begin{array}{l}\text { Participants : } \\
106 \text { délégués et observateurs venant } \\
\text { d'Europe, d'I Israesl et du Canada } \\
\text { (aucun pays du sud ni d'Asie). }\end{array}$ & $\begin{array}{c}\text { Participants : } \\
\text { Des délégués de } 12 \text { pays et } \\
\text { organisations internationales }\end{array}$ & $\begin{array}{c}\text { Participants : } \\
74 \text { délégués de } 10 \text { pays, } \\
\text { des observateurs de l' institut afro- } \\
\text { asiatique et } 6 \text { org. internationales } \\
\text { parmi lesquelles le Bureau } \\
\text { international du travail (BIT). }\end{array}$ & $\begin{array}{c}\text { Participants : } \\
\text { NR } \\
\text { 200 participants attendus } \\
\text { (Le Monde du Travail Libre }{ }^{\circ}{ }^{\circ} \text { 206) }\end{array}$ \\
\hline $\begin{array}{l}\text { Nombre de films proposés: } \\
\text { « tout un lot " }\end{array}$ & $\begin{array}{l}\text { Nombre de films proposés : } \\
\qquad 92\end{array}$ & $\begin{array}{c}\text { Nombre de films proposés : } \\
150 \text { (par } 19 \text { pays) }\end{array}$ & $\begin{array}{c}\text { Nombre de films proposés : } \\
135 \text { (18 pays) }\end{array}$ & $\begin{array}{l}\text { Nombre de films proposés: } \\
\text { NR }\end{array}$ \\
\hline $\begin{array}{l}\text { Nombre de films sélectionnés } \\
\qquad 26\end{array}$ & $\begin{array}{l}\text { Nombre de films sélectionnés } \\
\qquad 32\end{array}$ & $\begin{array}{l}\text { Nombre de films sélectionnés } \\
\qquad 8\end{array}$ & $\begin{array}{l}\text { Nombre de films sélectionnés } \\
47 \\
\text { (1 f film afficain pour la tase fois) }\end{array}$ & $\begin{array}{l}\text { Nombre de films sélectionnés } \\
\qquad 1 \text { (de } 14 \text { pays) }\end{array}$ \\
\hline $\begin{array}{l}4 \text { catégories } \\
\text { - Documentaires } \\
\text { - Films à scénario } \\
\text { - Films de propagande } \\
\text { - Actualités syndicales } \\
\text { hebdomadaires }\end{array}$ & $\begin{array}{c}9 \text { catégories } \\
\text { - Le Travail } \\
\text { - Fìms de fiction de court métrage } \\
\text { - Problèmes actuels } \\
\text { du mouvement ourrier } \\
\text { - Enseignement et éducation } \\
\text { démocratique } \\
\text { - Documents historiques } \\
\text { - Prévention d'accident et } \\
\text { protection de la santé } \\
\text { - Essais } \\
\text { - Propagande, } \\
\text { - L' 'ouvrier et l'art. }\end{array}$ & $\begin{array}{c}10 \text { catégories } \\
\text { - Le Travail } \\
\text { - Problèmes actuels } \\
\text { - Enseignement et éducation } \\
\text { démocratique } \\
\text { - Documents historiques } \\
\text { - Prévention d'accident et } \\
\text { protection de la santé } \\
\text { - Propagande } \\
\text { - L'ouvrier et l'art } \\
\text { - Réadaptation et orientation } \\
\text { professionnelles } \\
\text { - La Jeunesse } \\
\text { - Pays en voie de développement. }\end{array}$ & $\begin{array}{l}8 \text { catégories } \\
\text { - Les conditions du travail } \\
\text { - L'éduction syndicale } \\
\text { - Sujets syndicaux } \\
\text { - Problèmes sociaux } \\
\text { - Education et jeunesse } \\
\text { - Sécurité } \\
\text { - Problèmes nationaux } \\
\text { - Coopératives }\end{array}$ & $\begin{array}{l}5 \text { catégories } \\
\text { - Thèmes syndicaux } \\
\text { - Informations sociales } \\
\text { - Le bien-être du travailleur } \\
\text { - Problèmes économiques } \\
\text { et sociaux } \\
\text { - Edducation à la sécurité et } \\
\text { prévention des accidents }\end{array}$ \\
\hline $\begin{array}{c}\text { Film primé } \\
\text {-När ängarna blommar (Suède) }\end{array}$ & 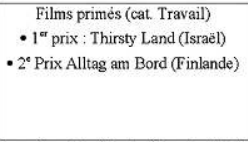 & $\begin{array}{l}\text { Films primés (cat. Travail) } \\
\text { - Counterpoint (Grande-Bretagne) } \\
\text { - Une casis dans le désert (Israell) }\end{array}$ & $\begin{array}{c}\text { Grand Prix } \\
\text { - Maximum (Suède) } \\
\text { Prix de la cat. Conditions du travail } \\
\text { - Phase delta I (Pays-Bas) }\end{array}$ & $\begin{array}{c}\text { Grand Prix } \\
\text { - Do not fold, staple or mutilate } \\
\text { (Canada) } \\
\text { Prix du jury } \\
\text { - The Inheritance (USA) } \\
\text { Les daux conceuraient dens la a 1"'ccateg. }\end{array}$ \\
\hline
\end{tabular}

Le tableau montre une difficulté des organisateurs à catégoriser les films des festivals, ainsi que les hésitations et les glissements qui se sont produits au cours des ans. Les 4 catégories originelles (du reste, assez floues) sont passées au nombre de 10 six ans plus tard. Celles-ci donnent une idée plus précise de l'éventail effectivement très large des films projetés. Il est intéressant de constater qu'une catégorie, «Le travail », est créée à Vienne en 1957, alors que l'intitulé même du festival (des films du travail) pouvait laisser entendre que tous les films présentés étaient censés y correspondre. Sous l'intitulé "Documents historiques ", qui apparaît également en 1957, il est probable qu'aient été projetés des moments choisis des conférences mondiales de la CISL, lesquelles étaient filmées. On peut le deviner à travers le commentaire excédé de Szombati qui, dans la préparation du festival de Vienne, dénonçait le fait de recevoir: " une assez grande quantité de bandes cinématographiques qui n'ont enregistré que des discours interminables » et qui n'étaient, selon lui, que « le triste témoignage d'un lamentable gaspillage d'argent ${ }^{37}$. "

Le resserrement des catégories à partir du festival de Tel-Aviv reflète le recentrage de l'activité de l'ILFI consécutif à la crise de 1961, sur des thématiques plus manifestement syndicales.

Ce tableau montre également qu'en fait d'internationalisation, les festivals de l'ILFI ne diffusent et ne couronnent guère que des films occidentaux, dont une grande majorité d'Europe du Nord. Il faut attendre le festival de Tel-Aviv ${ }^{38}$ en 1963 pour que l'internationalisation fasse un petit pas: pour la première fois un film africain fait partie de la sélection et l'institut afro-asiatique ${ }^{39}$ y envoie des observateurs (du Népal, Libéria, Japon, Kenya, Philippines, Ceylan). Il ne faut pas comprendre ici que la 
sélection aurait été excluante vis-à-vis de films non occidentaux. Tout simplement aucun (ou très peu à partir de 1963) n'était proposé. Cela reflète bien sûr la composition des membres de l'ILFI dont la provenance géographique a bien du mal à se diversifier. Les adhérents de l'ILFI resteront d'ailleurs en nombre très limité qui n'arrivera pas à augmenter au fil des ans. L'institut perd même des adhérents ${ }^{40}$ qui rechignent à payer deux adhésions (celle à la CISL et celle à l'ILFI).

L'impuissance de l'ILFI à se développer et à s'autofinancer constitue un échec important aux yeux de ses administrateurs. Il en découle des difficultés financières récurrentes qui auront progressivement raison de son autonomie.

Illustration 3. Image du film américain This Union Cause (1962).

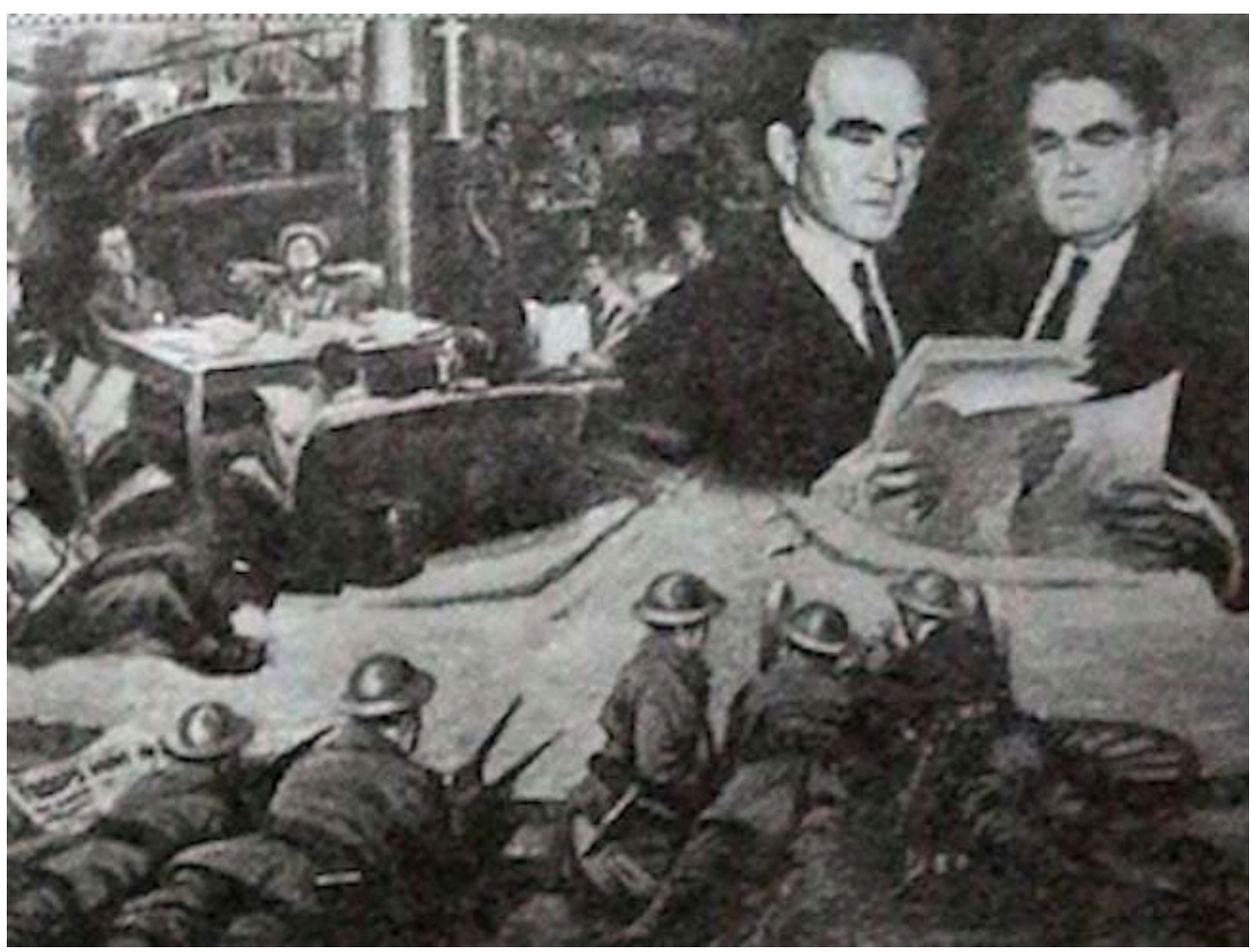

\subsection{Où sont les travailleuses ?}

Le jury du festival de Stockholm a "regretté l'absence de films sur la femme au travail ${ }^{41} »$ et aurait souhaité également recevoir davantage de films sociaux pour la télévision...

Il peut paraître étonnant qu'en 1960, l'oubli des femmes - dans les images ou ailleurs soit remarqué avant même d'être regretté : largement répandue, leur absence passe habituellement inaperçue. Il faut y voir plus qu'une coïncidence avec l'action très dynamique d'un groupe de femmes qui créent, dès 1953, le Comité des femmes de la CISL. Sigrid Ekendhal, syndicaliste suédoise, une de ses fondatrices, réussit à convaincre le Secrétaire général adjoint de la CISL Hans Gottfurcht de l'importance de ce comité et de la nécessité d'embaucher une permanente pour assurer son développement. Ce sera chose faite, précisément en mars 1957: Marcelle Dehareng, syndicaliste belge, est recrutée pour coordonner l'action du Comité des femmes, dont je détaille ailleurs les revendications en matière de formation et de représentation syndicale des femmes 
(Laot, 2015b). Pour autant, malgré l'appui de quelques-uns de ses responsables, la reconnaissance de ce comité tarde à venir au sein des instances de la CISL et il faudra attendre 1969 pour qu'une femme soit élue au comité exécutif. Ce sera la même chose pour l'ILFI. Les femmes y brillent par leur absence, jusqu'en... 1969, date à laquelle Marcelle Dehareng entre au Conseil d'administration. Elle y restera trois ans, mais dans une période de l'institut peu productive comme on le verra plus loin. Elle sera remplacée dans ses fonctions en 1972 par une autre femme, Adrienne Camusel qui n'aura guère le temps que de voter la dissolution de l'ILFI quelques mois plus tard. Deux ans auparavant toutefois, pour la première fois, une femme (sur 8 membres), participe au jury du festival de Montréal. Elle ne représente pas l'ILFI ni la CISL, mais l'Office national du film du Canada, co-organisateur de la manifestation.

Comme souvent, lorsqu'elles sont exclues des instances décisionnelles, les femmes ne sont pas pour autant éloignées du terrain. L'une d'elle, Renée Cahen, secrétaire administrative, portera l'ILFI à bout de bras pendant 3 ans. C'est elle qui sera la cheville ouvrière du festival de Tel-Aviv.

Photographie 3. Renée Cahen (secrétaire de l'ILFI) et Hans Saliger (Autriche) au festival de Tel-Aviv (1963).

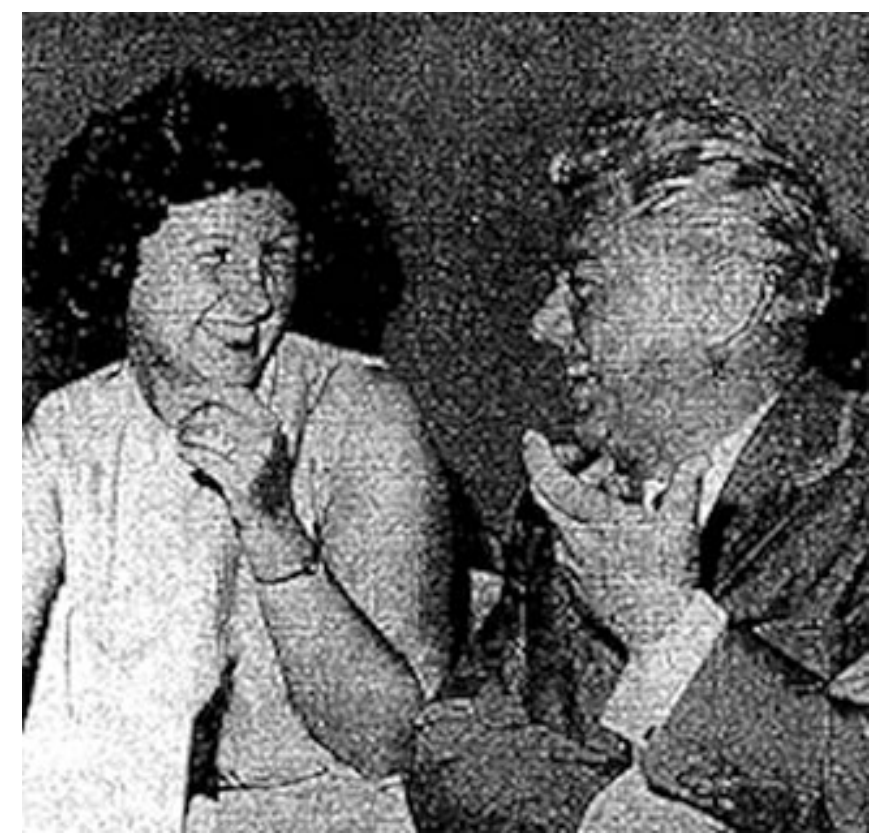


Illustration 4. Affiche du festival de Tel-Aviv.

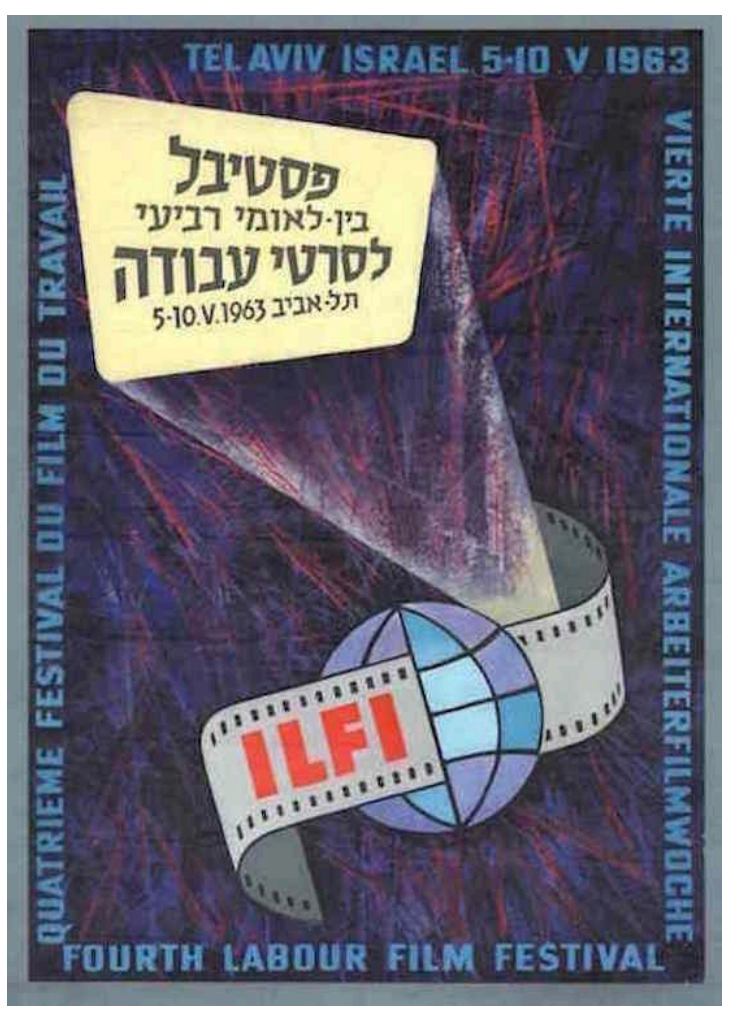

43 L'attention portée aux travailleuses grâce à l'action du Comité des femmes aura-t-il tout de même eu quelques effets sur le choix de quelques films?

Dans les listes de la cinémathèque de l'ILFI consultées dans les archives, deux ou trois documents portent à l'écran l'histoire de femmes, comme le film canadien de 1958, Women at Work ${ }^{42}$, qui montre le parcours de jeunes femmes venues d'Europe tenter leur chance au Canada, ou le film anglais de 1962, A Chance to Learn ${ }^{43}$, qui met en scène une veuve obligée de chercher du travail mais qui peine à en trouver car elle n'est pas qualifiée pour le maniement d'une machine. Ce film est en fait un plaidoyer pour la création de cours de formation professionnelle utiles à la fois aux employé-e-s et aux employeurs. Notons également la production, en 1965, d'un film fixe: Women's Role in Trade Unions directement lié au Comité des femmes. 
Photographie 5. Image d'un film en compétition au festival de Tel-Aviv.

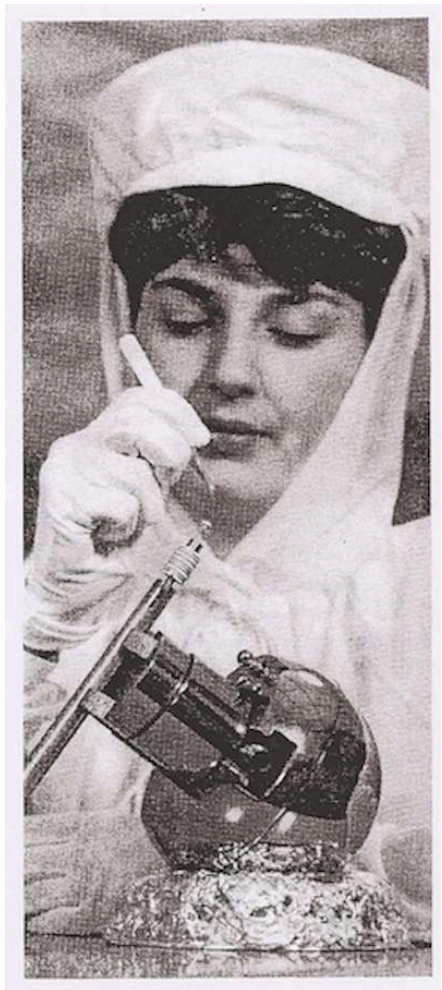

Photographie 6. Image du film Delta Phase 1 (1962) en compétition au festival de Tel-Aviv.

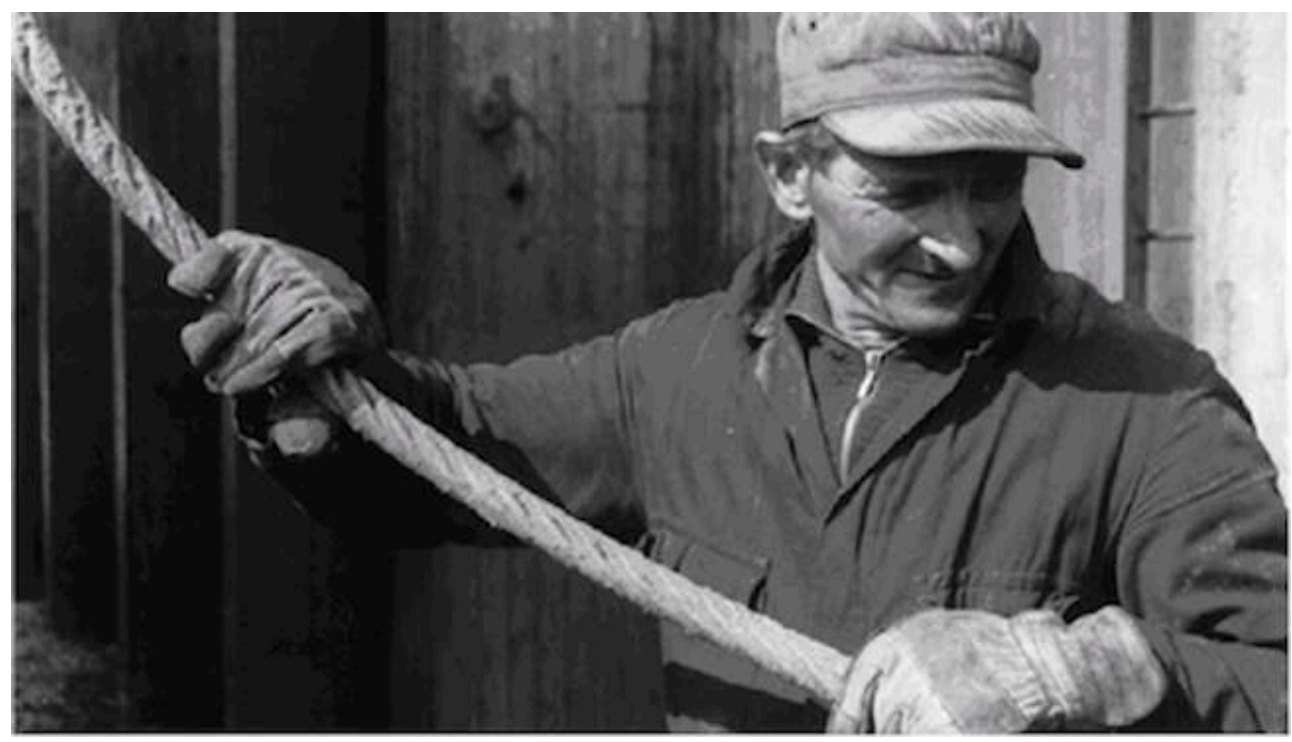

La thématique des femmes au travail dans les films répertoriés par l'ILFI reste exceptionnelle, la plupart montrent des univers d'hommes. Les plus demandés cependant (d'après le nombre de copies et de versions linguistiques ${ }^{44}$ ) montrent moins le travail que la lutte syndicale, en faisant la part belle à l'histoire du mouvement ouvrier. C'est le cas du film américain de 1962, This Union Cause ${ }^{45}$ qui retrace, à partir d'affiches, dessins et autres documents visuels, l'histoire de la classe ouvrière aux ÉtatsUnis et la création du premier syndicat. Il a eu le prix du meilleur film d'éducation syndicale à Tel-Aviv en 1963. Signalons également dans cette catégorie, le film Belge À 
l'Assaut du ciel ${ }^{46}$ qui relate « le plus important soulèvement populaire, la Commune de Paris et son influence sur le développement du mouvement ouvrier » ou encore le film américain de 1964 The Inheritance ${ }^{47}$, qui revisite cinquante ans d'histoire syndicale et politique des États-Unis. Selon Nathan Godfried (2014), qui en a fait une étude très poussée, ce dernier est tout à fait atypique dans la production filmique soutenue par les syndicats états-uniens, laquelle fait le plus souvent le jeu du capitalisme ${ }^{48}$. Il revient à une vision très sociale d'un syndicalisme de lutte qu'il relie aux problèmes contemporains que rencontrent les États-Unis en 1964, comme la question des droits civiques. En montrant une diversité de travailleurs - et de travailleuses - que l'on voit pour le coup au travail ${ }^{49}$, des migrant-e-s, des noir-e-s, les difficultés qu'ils et elles rencontrent au quotidien et les violences qu'ils et elles subissent ${ }^{50}$, il porte le message que la liberté reste un combat à gagner. Ce film a obtenu le prix du jury au festival de Montréal pour sa qualité artistique.

Illustration 6. Affiche du film The Inheritance (1964) primé à Montréal.

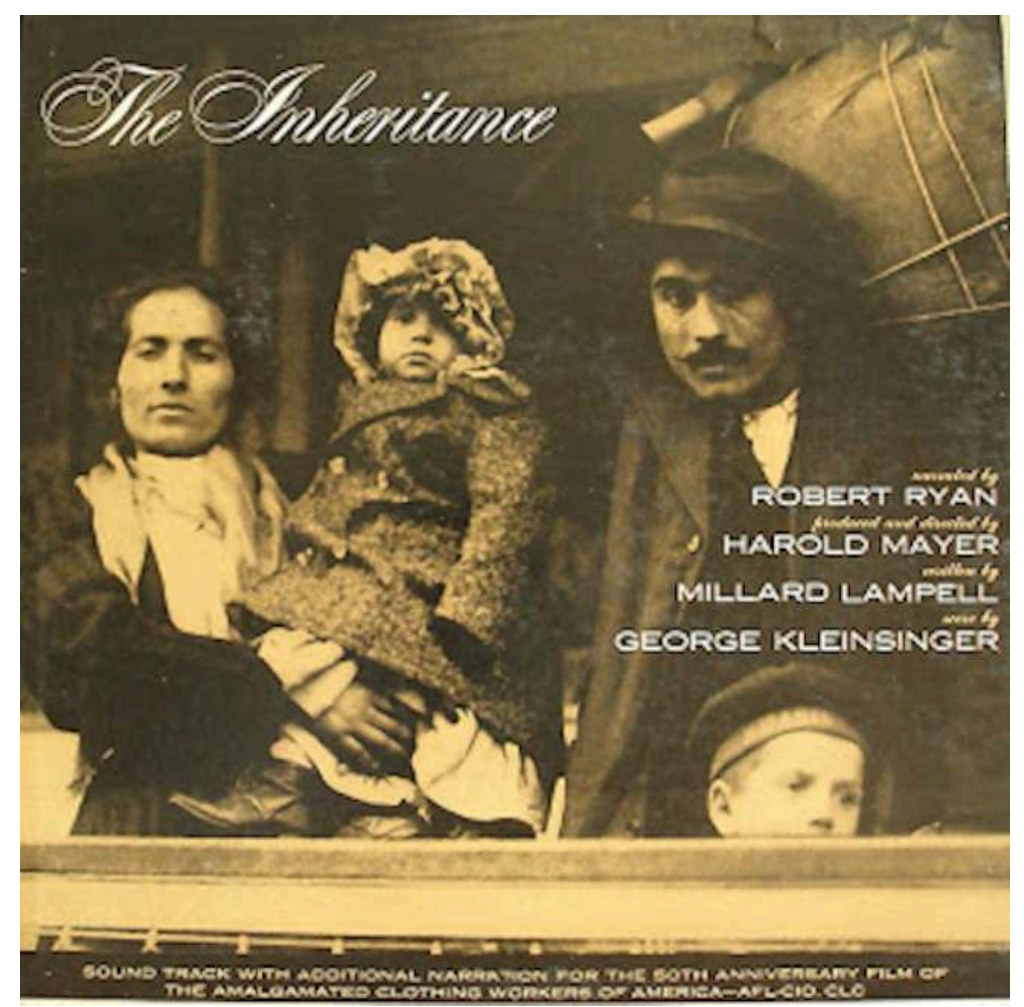

\section{Le film perd du terrain face à la montée de « l'audiovisuel » dans la formation}

\subsection{Réorganisation et recentrage de l'action de l'ILFI : un projet récurrent}

La crise financière de 1961 a mis l'ILFI un peu plus sous la tutelle de la CISL. Après le départ de Szombati, le poste de direction reste vacant. Renée Cahen est réembauchée par la CISL et mise à disposition de l'ILFI. Mais elle démissionne, pour des raisons personnelles, en 1964. Son départ non anticipé plonge les administrateurs dans 
l'embarras. Ils cherchent, visiblement sans grand succès, de nouveaux animateurs pour l'ILFI. En octobre 1964, l'institut est intégré au département Éducation, Femmes et Jeunesse de la $\mathrm{CISL}^{51}$, son siège placé dans ses locaux et ses frais généraux pris en charge par la confédération. Le fonds de solidarité de la CISL, créé pour financer les actions à mener en direction des pays en voie de développement, contribue au financement de l'ILFI en complément de l'aide financière de l'UAW qui représente près de la moitié du budget total ${ }^{52}$. Il n'est donc pas exclu que l'ILFI ait, de ce fait, bénéficié de fonds de la $\mathrm{CIA}^{53}$, cette information n'ayant toutefois pas pu être vérifiée dans les documents consultés.

Juridiquement, l'ILFI reste pourtant une association "indépendante », mais qui "par suite de circonstances diverses [...] n'a pu prendre de grandes initiatives ces derniers mois ${ }^{54}$. Maintenir une structure associative permet de garder la FIAMP en son sein. De nombreux documents d'archives laissent entrevoir que l'ILFI essuie de nombreuses critiques et rapportent l'insatisfaction de ses administrateurs quant à son fonctionnement. Les membres du conseil se renouvellent souvent. Louis Major se maintient toutefois à son poste de président, voulant croire que l'institut pourra continuer à « faire le job pour lequel il a été créé ${ }^{55}$. " Malgré cette morosité, le modèle s'exporte : un $1^{\text {er }}$ festival des films du travail est organisé aux États-Unis en 1964, à l'issue duquel est créé un «Institut américain des films du travail ».

En 1965, Paul Louyet, directeur des services cinématographiques du ministère belge de la Culture fait de nouvelles propositions d'organisation pour l'ILFI qui l'amènent à en devenir le "directeur technique $»^{56}$. Il s'agit en particulier de créer un réseau de correspondants dans les différents pays membres et de réorganiser la cinémathèque afin d'en faire l'activité centrale de l'institut (augmentation des collections, documentation sur les films et prêt). Les correspondants ont pour mission de faire le lien entre les productions et les besoins de leur pays et l'ILFI. Une liste de correspondants pour 14 pays membres (tous européens sauf les États-Unis, le Canada et Israël), est établie mais avec de grandes disparités : trois noms de correspondants y figurent pour l'Autriche, l'Allemagne et les États-Unis, tandis que pour cinq autres pays, dont la France, aucun nom n'est inscrit. En outre, ce dispositif semble avoir mal fonctionné. En effet, quelques mois plus tard, une lettre de Louyet aux correspondants montre son mécontentement: "la grande majorité n'a même pas pris le temps de répondre " à ses sollicitations ou alors « hors délais raisonnables ». Il ajoute qu'il « est inutile d'attendre des résultats positifs de l'ILFI sans la collaboration des pays membres $^{57}$.».

L'ILFI continue pourtant son travail de fond: la synchronisation de nouveaux films, la production de films fixes, l'administration de la cinémathèque. Il recentre son action sur des films plus ostensiblement « syndicaux»:

« Nous avons acquis un stock de films traitant de sujets variés (sécurité, hygiène, formation professionnelle,...) mais nous nous sommes rendus compte que nous redoublions ce que faisaient déjà des organisations nationales [...] En conséquence, nous avons modifié notre politique pour nous consacrer davantage à la diffusion de films sociaux traitant de différents aspects de la lutte syndicale, pour des raisons d'ordre pratique mais aussi pour des raisons plus profondes. Nous pensons qu'il est bon de travailler à la cause syndicale, de travailler à augmenter la claire conscience de la part des travailleurs, par le moyen de films les amenant à se former une opinion en pleine conscience des enjeux et de cristalliser leurs vues par rapport aux problèmes sociaux en général. Nous sommes heureux de dire que cette nouvelle politique a soulevé un très grand enthousiasme parmi les pays membres de notre 
institut [...], nous avons pu constituer une cinémathèque qui, nous l'espérons, va se développer rapidement ${ }^{58}$. "

Le contexte joue manifestement dans ces réorientations. Dans le milieu des années 1960, les deux grandes confédérations, la CISL et la FSM, traversent une phase délicate d'adaptation à une situation internationale qui s'est modifiée avec le dégel des deux blocs. Le président de l'AFL-CIO ${ }^{59}$ états-unien, qui veut maintenir coûte que coûte un anticommunisme pur et dur, n'est plus suivi. Omer Becu, Secrétaire général de la CISL depuis 1961, dans son rapport au congrès écrit que « la CISL n'est pas un instrument de la guerre froide, elle n'est d'aucun bloc ou gouvernement » (Sicinius, 1965, 518 ; Carew, $2000,301)$. Dans le "camp d'en face ", la FSM se divise également entre une ligne antiimpérialiste dure (portée par les chinois) et une orientation plus ouverte (portée par les italiens). Son unité se fractionne à travers différents conflits entre 1964 et 1968, date de la répression du printemps de Prague qui soulève la réprobation de nombreux membres (Devinatz, 2013). En outre, la Communauté économique européenne en construction pousse les deux OSI à adopter une articulation régionale. Elles se rejoignent donc partiellement sur l'idée d'une coexistence pacifique. Cette évolution les pousse à se recentrer sur une activité plus clairement syndicale que politique. On voit la trace de cet infléchissement dans les orientations de l'ILFI à travers les films à privilégier.

En 1966, un cinquième festival est en préfiguration. Il sera retardé d'un an et aura finalement lieu à Montréal grâce, encore une fois, à une subvention de l'UAW. Mais l'ILFI n'en est plus le seul porteur, il en partage l'organisation avec l'office national du film du Canada et le Congrès du travail du Canada. Notons de plus que parallèlement à ce festival est organisé un symposium sur l'utilisation des moyens audiovisuels.

Photographies 7 et 8 . Images de films en compétition au festival de Montréal (1967).

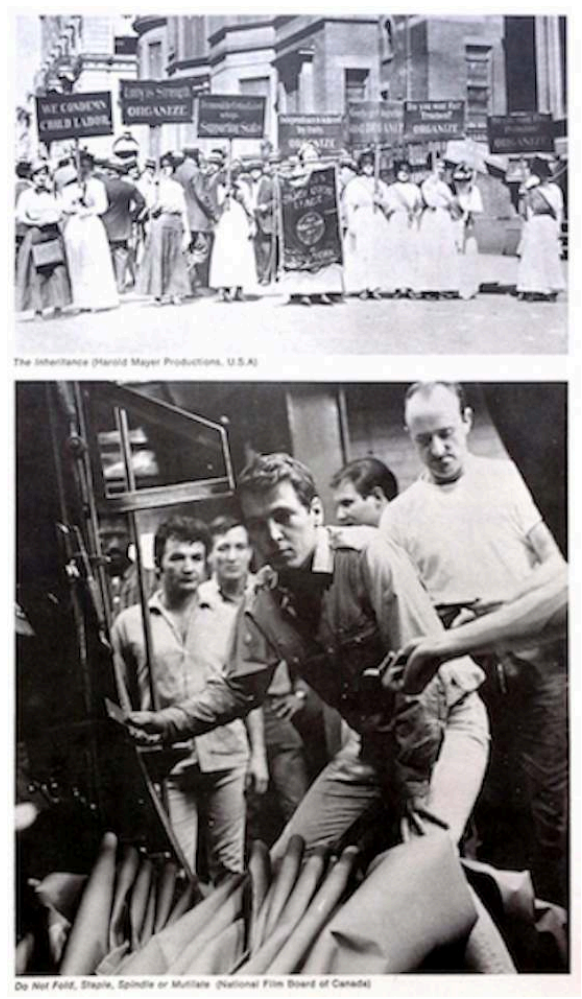




\title{
3.2. Des « moyens audiovisuels » de formation moins coûteux et plus souples d'utilisation
}

\begin{abstract}
«L'expression "moyens audio-visuels" peut paraître assez compliquée et technique. En fait, c'est seulement une locution moderne de moyens d'expression vieux comme le monde. On appelle en effet "audio-visuel" tout ce qui appartient à la méthode active d'enseignement qui utilise au maximum les sens de l'être humain, et en particulier l'ouïe et la vue. Les premiers chants, les dessins primitifs gravés sur les mur des grottes préhistoriques, les rythmes de la danse, du drame, de la composition et des travaux manuels, les couleurs, le langage parlé et écrit étaient les moyens audio-visuels d'expression et de communication dont se servaient les hommes et les femmes aux premiers temps de l'histoire. Ces moyens traditionnels existent encore de nos jours ${ }^{60}$. »
\end{abstract}

On peine à croire, aujourd'hui, qu'il faille définir «les moyens audiovisuels » dans la brochure éponyme éditée par la CISL en 1964, tout en soulignant leurs côtés "compliqués et techniques". Les films sont bien entendu inclus, parmi beaucoup d'autres "moyens", dans cette brochure où il est souligné qu'ils exercent un effet «puissant sur un public nombreux ». Leur utilisation, cependant, est présentée comme soulevant de sérieux inconvénients : les bons films sont rares, le matériel coûteux à acheter et à entretenir, d'emploi compliqué... de plus, sauf lorsqu'ils sont correctement employés, les films ne permettent qu'une communication à sens unique. Leur utilité est accrue lorsqu'ils sont utilisés dans des groupes de discussion. Quoi qu'il en soit, il y a de "gros efforts à faire pour obtenir la production de bons films ${ }^{61}$.» En comparaison, les films fixes sont qualifiés de relativement peu coûteux et d'emploi facile. Les autres «moyens audiovisuels » comme la radio, la télévision, le tableau noir [sic] ou de feutre, les journaux muraux sont présentés de même selon leur coût et leur degré d'accessibilité, tous plus faciles d'utilisation que les films.

Les années qui suivent sont celles du plein développement des médias (TV) et de nouveaux supports, les films super-8, les Mini-Cassettes audio et les premiers magnétoscopes... En parallèle la formation permanente s'institutionnalise dans la plupart des pays occidentaux. Avec elle, la réflexion pédagogique bat son plein.

Face à ces deux mouvements conjugués, l'ILFI adapte son action. Il s'oriente progressivement vers la production de diapositives et de films fixes et même vers l'édition de disques de chants du travail. Il développe ensuite des outils pour la formation de formateurs en constituant des dossiers sur l'utilisation de moyens pédagogiques et d'«accessoires didactiques » combinant feuillets mobiles, films fixes, bandes magnétiques, présentation de cas, exercices, jeux de rôles...

La formation syndicale elle-même est en pleine transformation (Éthuin, Yon, 2014). Depuis l'origine préoccupée par ce thème, la CISL organise sa première Conférence mondiale sur l'éducation et le mouvement syndical à Montréal dans les jours qui suivent le festival des films du travail. On y repense le rôle des syndicats dans l'élaboration des politiques publiques d'éducation. On y insiste sur la nécessité du congé-formation pour permettre aux travailleurs de "poursuivre leur éducation au cours de leur vie laborieuse ${ }^{62}$. $"$ Herbert Tulatz, secrétaire adjoint de la CISL, organisateur de la conférence et secrétaire de l'ILFI, y déclare : «dans le domaine de l'éducation syndicale, cette conférence marque le tournant d'un mouvement syndical fidèle à la tradition s'adaptant à l'ère de la technologie ${ }^{63}$. " Ces prises de position influenceront les actions de l'ILFI. 


\subsection{II n'y aura pas de $6^{\mathrm{e}}$ festival : I'ILFI en capilotade}

traverser une nouvelle période difficile en raison d'une succession d'épreuves. Herman Patteet, son trésorier, est arrêté et mis en examen pour détournement de fonds, ce qui jette la suspicion sur les comptes de l'ILFI pour la période comprise entre le $1^{\text {er }}$ juin 1965 et le 31 décembre 1967. Herbert Tulatz décède en juin. Enfin, le 8 octobre, Louis Major, nommé ministre belge de l'emploi et du travail envoie sa lettre de démission. À la fin de l'année, il ne reste quasiment plus à l'ILFI que le directeur technique, Paul Louyet. On peut ajouter à cette suite funeste, la situation guère plus brillante de la CISL. Elle connaît elle-même une crise majeure ${ }^{64}$ qui conduit au départ des états-uniens en 1969 et met en jeu son existence même (Carew, 2000).On peut comprendre que ce départ entraîne de nouvelles difficultés financières pour l'institut. Son Conseil d'administration est entièrement renouvelé en juillet 1969, mais l'activité reste réduite.

LAssemblée générale de février 1970 décide de repousser à 1971 le $6^{e}$ festival des films du travail et élit au poste de Secrétaire général Daniel Pedersen, le successeur de Tulatz au département Éducation de la CISL. Celui-ci ne reprend pas le projet du $6^{e}$ festival et organise à la place deux séminaires internationaux sous l'égide du l'ILFI qu'il dirige luimême. Le premier, a lieu en avril 1971 au Danemark. Il est consacré aux « Moyens audiovisuels pour la formation syndicale ». L'objectif est d'encourager leur utilisation dans la formation syndicale, à partir d'une démonstration de matériels tels que les vidéocassettes et la télévision en circuit fermé. L'envergure de cette manifestation s'avère très modeste puisqu'elle ne réunit que quelques " éducateurs » de 14 pays. Le deuxième séminaire a lieu en avril 1972 en Suisse, sur le thème : " Matériel didactique pour la formation syndicale ». Les moyens audiovisuels n'y sont même plus l'objet central. On peut se demander en quoi il était indispensable d'inscrire cette activité au sein d'un Institut international des films du travail. D'ailleurs est-il encore utile à quelque chose ? L'Assemblée générale de 1972 met en place un groupe de travail chargé « d'étudier la portée des activités et les possibilités économiques de continuer l'ILFI en tant qu'entité séparée ou bien de le dissoudre pour intégrer ses fonctions au Département d'éducation de la CISL ${ }^{65}$. »

Une Assemblée générale extraordinaire, de taille réduite puisqu'elle réunit moins d'une dizaine de personnes représentant autant d'institutions, vote en effet la dissolution le 23 novembre 1972. Le liquidateur établit au 31 décembre de la même année l'inventaire des biens de l'ILFI, comprenant pour l'essentiel du matériel de projection et des films. Le tout est remis à la CISL qui s'engage à continuer son action. L'institut n'est plus.

\section{Conclusion : Des films du travail... sans travail ?}

Le projet de l'ILFI était ambitieux, son action semble avoir pourtant toujours été cantonnée dans des dimensions très modestes, freinée par de nombreuses difficultés récurrentes. A-t-il jamais connu une période faste? Même si deux institutions étaient officiellement à l'origine de sa création, l'ILFI ne parviendra jamais vraiment à s'émanciper de la CISL. Dépendant financièrement d'un gros syndicat états-unien, il n'a pas su garder ses personnels et n'a jamais réussi à augmenter le nombre de ses adhérents. Il a pourtant travaillé, pendant 20 ans, à organiser une cinémathèque, à

Images du travail, travail des images, 2 | 2016 
réaliser différentes productions (dont un seul film), à inciter les syndicats des différents continents à se former à travers les films et à organiser 5 festivals internationaux. L'idée de former, par l'image et le son, les syndicalistes et les formateurs de syndicalistes de tous les pays est restée son but premier. En ce qui concerne les festivals, on peut toutefois s'interroger sur leur pertinence et leur portée réelle. Chaque édition semble en effet n'avoir rassemblé qu'un nombre limité de participants, sans doute moins de 200 .

Au-delà de l'intérêt porté à la vie d'un institut, aussi singulier soit-il, retracer son action nous a permis de revisiter une période très riche en matière d'éducation liée à l'image. Dans les premières années, la force des films, leur valeur éducative, leur pouvoir d'influence, étaient incontestés. Au fil des ans, d'autres «moyens » ont peu à peu éclipsé leur suprématie.

61 L'histoire de l'ILFI constitue enfin une entrée nouvelle pour étudier l'histoire du mouvement syndical mondial et de la CISL en particulier. Les évolutions et les adaptations de la confédération dessinent, en parallèle, la destinée de l'ILFI. Cette étude apporte une petite pierre complémentaire : l'institut n'est pas cité dans le gros volume consacré à l'histoire de la CISL dirigé par van der Linden (2000). Il serait intéressant d'aller rechercher si la FSM faisait autant de cas des films et de leur utilisation dans la formation syndicale qui était, pour elle aussi, considérée comme une partie centrale de l'action syndicale internationale.

Quant aux films promus à travers cette expérience, ils mériteraient une recherche à part entière. La cinémathèque comptait environ 250 films au plus fort de son activité. Plutôt qu'à des « films du travail », ils s'apparentent à des films "d'intérêt syndical », comme ils ont pu être qualifiés parfois à l'ILFI. Reste à vérifier si les hommes - et les femmes - dans ces documents-là, ont été portés à l'écran au travail, car, comme il est souligné de manière critique par les représentants de l'ILFI, invités à la quatrième Triennale du film pour le travail et l'industrie à Anvers en 1963: "parmi les films projetés, il a été constaté que le travailleur est souvent peu représenté dans le cadre qui lui est familier; on s'attarde volontiers sur les machines, leur complexité, l'étendue de l'entreprise, mais l'homme est généralement $a b s e n t{ }^{66}$. ». Les films de la cinémathèque de l'ILFI auraient-ils, plus que d'autres à la même époque, montré le travail ?

\section{BIBLIOGRAPHIE}

Borde R. et Perrin C. (1992), Les Offices du cinéma éducateur ou la survivance du muet, 1925-1940, Lyon, Presses universitaires de Lyon.

Carew A. (2000), “Towards a Free Trade Union Centre: The International Confederation of Free Trade Union (1949-1972)", in Van der Linden M. (dir.), The International Confederation of Free Trade Unions, Bern: Peter Lang, p. 187-339.

Devinatz G. V. (2013), “A Cold War Thaw in the International Working Class Movement? The World Federation of Trade Unions and the International Confederation of Free Trade unions, 
1967-1977", Faculty Publications-- Management and Quantitative Methods [En ligne], 7-2013, mis en ligne le 23 janvier 2014.

URL : http://ir.library.illinoisstate.edu/fpmqm/7

Espéret G. (1965), « L'évolution actuelle du syndicalisme international », Politique étrangère, vol. $30, n^{\circ} 4-5$, p. 444-462.

Éthuin N. et Yon K. (dir.), 2014, La fabrique du sens syndical. La formation des représentants des salariés en France (1945-2010), Bellecombes-en-Bauge, Ed. du Croquant.

Godfried N. (2014), “Labor sponsored Film and Working-Class History: The Inheritance (1964)", Film History: An International Journal, Volume 26, Number 4, p. 84-119.

Goutanier C. et Lepage J. (2008), « Le film fixe : une source à découvrir. Un exemple de sauvegarde en Anjou », Histoire@Politique, Culture, Société, n² 4, p. 18-18.

Hatzfeld N. (2013), « Figures filmiques d'ouvrières : travail, genre et dignité, variations sur une trilogie classique (1962-2011)», Clio. Femmes, Genre, Histoire, n 38, p. 79-96.

Hatzfeld N., Rot G. et Michel A. (2009), « L'ouvrier en personne, une irruption dans le cinéma documentaire (1961-1974)», Le Mouvement Social, n² 226, p. 67-78.

Jarry É. (2001), «L'aventure de la coopérative du Cinéma du Peuple », Le Monde Libertaire, $\mathrm{n}^{\circ} 1251$ [En ligne], consulté le 5 mars 2015.

URL: http://web.archive.org/web/20051018042030/http://www.federation-anarchiste.org/ml/ article.php3?id_article $=2825$

Laborderie P. (2015), Le cinéma éducateur laïque, Paris, L'Harmattan.

Laot F. F. (2015), « Un ouvrier "burlesque” a-t-il sa place dans le cinéma éducatif syndical ? Débats autour d'un film de l'Institut international des films du travail (1958-1965) », dans Rochet B., Bettens L., Gillet F., Machiels C. et Roekens A. (dir.), Quand l'image (dé)mobilise. Iconographie et mouvements sociaux au XX $X^{e}$ siècle, Namur, Presses Universitaires de Namur, p. 167-177.

Laot F. F. (2015), « La formation des travailleuses : une revendication du syndicalisme mondial? Contribution à une histoire dénationalisée de la formation des adultes (1950-1968) », Le Mouvement social, $\mathrm{n}^{\circ}$ 253, p. 65-87.

Perron T. (1995), « "Le contrepoison est entre vos mains, camarades”. CGT et cinéma au début du siècle ", Le Mouvement Social, $\mathrm{n}^{\circ}$ 172, p. 21-37.

Perron T. (2000), «À la recherche du cinéma ouvrier : périodisation, typologie, définition », Les cahiers de la cinémathèque, $\mathrm{n}^{\circ} 71, \mathrm{p} .9-14$.

Ory P. (1975), « De “Ciné-Liberté” à La Marseillaise. Espoirs et limites d'un cinéma libéré (1936-1938) », Le Mouvement Social, $\mathrm{n}^{\circ}$ 91, p. 153-176.

Rochet B., Bettens L., Gillet F., Machiels C. et Roekens A. (dir.), 2015, Quand l'image (dé)mobilise. Iconographie et mouvements sociaux au XXe siècle, Namur, Presses Universitaires de Namur.

Sicinius. (1965), « Orientation nouvelles dans le mouvement syndicaliste », Politique étrangère, $\mathrm{n}^{\circ} 6, \mathrm{p} .517-523$.

Sorlain Pierre (coord.), 1995, Cinéma : le premier siècle ; CGT : le premier siècle, Le Mouvement social, $\mathrm{n}^{\circ} 172$, p. 3-108.

Taillibert C. (1998), « Le cinéma, instrument de politique extérieure du fascisme italien », Mélanges de l'École française de Rome, Italie et Méditerranée, tome 110, n², p. 943-962. 
Tartakoswki D. (1996), « Les centenaires croisés du syndicalisme et du cinéma », Vingtième Siècle. Revue d'histoire, $\mathrm{n}^{\circ}$ 50, p. 140-143.

Tartakoswki D. (1996), «Les centenaires croisés du syndicalisme et du cinéma », Vingtième Siècle. Revue d'histoire, $\mathrm{n}^{\circ}$ 50, p. 140-143.

Van der Linden M. (Ed.), 2000, The International Confederation of Free Trade Unions, Bern: Peter Lang. Van der Linden M. (2012), « Enjeux pour une histoire mondiale du travail », Le Mouvement social, n' 241, p. 3-29.

Vignaux V. (2007), Jean Benoit-Lévy ou le corps comme utopie. Une histoire du cinéma éducateur dans l'entre-deux-guerres en France, Paris, AFRHC.

Windmuller P. J. (1961), “ICFTU after Ten Years”, Industrial and Labor Relations Review, vol. 14, n², p. 257-272.

\section{NOTES}

1. International Institute of Social History. Ces archives sont tantôt uniquement en anglais, tantôt également en français, parfois en allemand. International Labour Film Institute archives : 1503-1512 ; 2527-2547. International Institute of Social History. Ces archives sont tantôt uniquement en anglais, tantôt également en français, parfois en allemand. International Labour Film Institute archives : 1503-1512; 2527-2547.

2. Celui de la CGT qui dispose d'un petit fonds d'archives de la Fédération syndicale mondiale, de la CFDT qui contient des documents en lien avec la Confédération internationale des syndicats chrétiens, mais également ceux de l'Unesco à Paris et du BIT à Genève.

3. Ou labor en anglais américain.

4. Alexandre Szombati a été directeur de l'ILFI de 1957 à 1961. Szombati A. (1957), « Le film et le mouvement ouvrier ", Le Monde du travail libre, $\mathrm{n}^{\circ} 85$, p. 42.

5. Aujourd'hui encore, le secteur vidéo de la CNT, Caméra au poing, publie sur son site un catalogue de films intitulé «Syndicalisme, monde ouvrier et cinéma » : Antony M. et coll., mise à jour 2011, "Syndicalisme, monde ouvrier et cinéma», http://www.cnt-f.org/video/images/ stories/pdf/syndicalisme-monde-ouvrier-et-cinema-22sept2011.pdf.

6. Deutscher Gewerkschaftsbund.

7. Reuter G. (1954), «Le premier festival international du film ouvrier », Le Monde du travail libre, $\mathrm{n}^{\circ} 53$, p. 23.

8. En 1959, 45,4 \% de adhérents sont européens, 26,2 \% nord-américains, 11,3\% d'Amérique du Sud, $11,2 \%$ d'Asie tandis que ceux d'Afrique représentent à peine 2,3 \% et le Moyen-Orient (en fait Israël), 1,3 \% (Windmuller, 1961).

9. C'est un « rouleau de pellicule transparente et noire de $35 \mathrm{~mm}$, d'une longueur d'un mètre environ qui comporte des vues en noir et blanc parfois avec de la couleur. Il est composé de photos (surtout pour les films d'enseignement général), de dessins (surtout pour les films récréatifs et religieux), de cartes parfois accompagnées de textes. » (Goutanier et Lepage, 2008).

10. C'était le candidat des syndicalistes anglais, mais les américains ont bloqué sa candidature (Carew, 2000).

11. (1896-1982), Allemand réfugié au Royaume-Uni pendant la guerre. Responsable du département de l'Éducation et Secrétaire général adjoint de la CISL (1952-1959).

12. (1914-1968), Allemand. Secrétaire général adjoint de la CISL.

13. Syndicaliste danois. 
14. Rapports d'activité et financiers, 1957-1959, 6 e Congrès, CISL, Bruxelles, 3-12 décembre 1959, p. 161. En fait, en difficulté à la tête de la CISL, il sera "débarqué » de son poste de Secrétaire général par les américains en 1960 et remplacé par Omar Becu (Windmuller, 1961).

15. Dont les Amis du Parti socialiste français qui adhérera en 1965.

16. IISH - ILFI Archives 1503-1504.

17. Vraisemblablement sur les conseils des représentants de la FIAMP.

18. Reuter G. op.cit. p. 28.

19. En 1961, il y obtiendra le «Statut de membre de catégorie A ». Rapport sur le 8 e Congrès mondial de la CISL, Amsterdam, 7-15 juillet 1965, p. 276.

20. Catalogue international de films du travail, ILFI, 1956, p. V.

21. Sujets syndicaux d'un intérêt général/syndicats particuliers - par industrie/problèmes du travail, conditions de travail et sujets similaires/éducation et jeunesse ouvrière/sujets sociaux et économiques, compréhension internationale/productivité, sécurité du travail, éducation professionnelle/coopératives, maisons du peuple et sujets similaires/congés, voyages et sports ouvriers/films à scénario, basés sur des sujets ouvriers ou similaires.

22. La plupart des autres syndicats membres de la CISL n'ont pas connu de scission. La CGT française et la CGIL italienne sont les deux seuls syndicats du monde occidental à être restés membres de la FSM qui réunit tous les syndicats du monde communiste.

23. Rose Étienne entre au Bureau confédéral de la CGT-FO en 1948. Elle y est alors la seule femme. Elle s'y occupe d'abord de l'ensemble des questions relatives à l'enseignement et à l'éducation ouvrière ainsi que des questions féminines. Elle prend la responsabilité de l'éducation ouvrière à la confédération de 1948 à 1961 où elle est également responsable de la section cinéma. Notons que contrairement à la CGT et à la CFDT, les archives de la CGT-FO n'ont pas été centralisées ni organisées. Elles n'ont donc pas pu être consultées.

24. Elle est membre du Conseil consultatif.

25. Catalogue... op.cit. p. VII.

26. Préambule aux statuts de la CISL et exergue à l'article : Gottfurcht H. (1951), « La conférence européenne sur l'éducation ouvrière organisée par la CISL », Les Cahiers de Fernand Pelloutier, $\mathrm{n}^{\circ}$ 15, p. 7-8.

27. International Centre of Worker's Education, Meeting of Experts, 8-15 August 1953, Unesco, Unesdoc (citation traduite).

28. Propos prêtés à Gottfurcht par Szombati A. (1960), «Le triomphal Festival des films du travail », Le Monde du travail libre, $\mathrm{n}^{\circ}$ 121, p. 315.

29. IISH-ILFI Archives, Gottfurcht H., The Labour Movement and the Film, Lecture, $3^{\text {rd }}$ ILFI Festival. Stockholm, 22-27 May 1960.

30. Ibid.p. $2 ; 8$.

31. Ibid. p. 5.

32. Hongrois réfugié en Belgique pendant la guerre. Il restera quatre ans et demi à la tête de l'ILFI, de 1957 à 1961.

33. Il est directeur du service de l'Éducation et de la Culture de Histadrout (Fédération générale des travailleurs de la Terre d'Israël).

34. Joseph Burstein, cité par Szombati A. « Le triomphal festival... », art. cit. p. 315-316.

35. Ibid.

36. IISH-ILFI Archives (ICFTU-CISL). Note confidentielle envoyée aux membres du CA de l'ILFI en novembre 1962.

37. Szombati A., «Le film et le mouvement ouvrier », op.cit., p. 43.

38. Israël était un des rares pays affiliés représentant le « Moyen-Orient ».

39. Dès sa constitution, la CISL tente, non sans mal, de mettre en place des organisations régionales qui couvrent tous les continents.

40. Il n'y a que 19 organisations affiliées en 1969 (20 en 1968). 
41. Szombati A., «Le triomphal festival du film du travail », op. cit., p. 317.

42. $21 \mathrm{~min}$, noir et blanc, office national du film, version anglaise, néerlandaise et allemande.

43. Fred Moore, anglais, $20 \mathrm{~min}$, noir et blanc.

44. Figurant dans les listings de films dans les archives de l'ILFI.

45. $23 \mathrm{~min}$, couleur. Film produit par la United Automobile Workers of America.

46. 1966, Jan Peré, 20 min, film tourné en néerlandais, mais avec des versions anglaise, française, allemande et espagnole, ministère de l'Éducation nationale et de la Culture belge.

47. Harold Mayer, USA, noir et blanc, $55 \mathrm{~min}$, produit avec le soutien du Amalgamated Clothing Workers of America (syndicat des ouvriers du textile des États-Unis).

48. Georges Meany, le président de la fédération américaine AFL se vantait de n'avoir jamais participé à une grève de sa vie (Godfried, 2014, 87).

49. Mais dans des séquences très courtes en succession rapide (le film est construit sur le rythme et la musique).

50. Il rend en particulier un hommage à Ida Brayman, une jeune migrante de 17 ans, tuée par un commerçant alors qu'elle incitait, avec d'autres syndicalistes, les travailleurs à rejoindre leur grève en 1913.

51. On notera au passage tout l'intérêt de cet intitulé...

52. Selon Reuther, l'UAW a financé l'ILFI a hauteur de 100000 dollars. IISH, Correspondance de l'ILFI, Lettre de Reuther à Becu du 18 octobre 1966. En 1968, la contribution de l'UAW représente 45,3\% du budget tandis que l'autofinancement de l'ILFI représente $13,5 \%$ seulement.

53. En effet, la CIA a financé les syndicats états-uniens pour leurs actions extraterritoriales, bien que leurs leaders se soient défendus de n'en rien savoir, ce que Carew (2000) juge plausible concernant Walter Reuther.

54. IISH-ILFI Archives, Note préparatoire à la réunion du Conseil d'administration du 10 octobre 1964.

55. IISH-ILFI Archives, Assemblée générale du 9 mars 1965.

56. Néanmoins, les archives ne permettent pas d'établir s'il s'agit d'un poste rémunéré. On retrouve en effet Louyet par la suite dans le Conseil de direction de l'ILFI où il remplace W. Schevenels.

57. IISH-ILFI Archives, correspondance de ILFI, Lettre de Paul Louyet aux membres correspondants, 14 janvier 1966.

58. IISH-ILFI Archives, Point sur l'action de l'ILFI par rapport aux mandats confiés par la CISL. Note du trésorier Patteet, $1^{\mathrm{er}}$ avril 1967.

59. American Federation of Labor and Congress of Industrial Organizations.

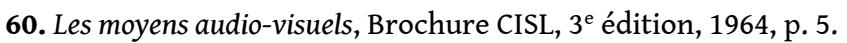

61. Ibid. p. 49.

62. "Les syndicats et l'éducation. Montréal ouvre une voie nouvelle », Le Monde du travail libre, $\mathrm{n}^{\circ} 208$, octobre 1967, p. 5.

63. Ibid. p. 4.

64. Celle-ci tient en en grande partie au refus des représentants de l'AFL CIO de la politique d'ouverture de la CISL consistant à tolérer les contacts avec des organisations communistes (Carew, 2000).

65. IISH. ILFI Assemblée générale du 8 juillet à Londres.

66. Rapport sur le $8^{\mathrm{e}}$ congrès mondial, Amsterdam 7-15 juillet 1965, CISL, p. 281 (chapitre consacré à l'ILFI). 


\section{RÉSUMÉS}

L'Institut international des films du travail, l'ILFI (International Labour Film Institute), issu du mouvement syndical mondial, a consacré son activité à l'éducation ouvrière par le cinéma. Les conditions de sa création et son action au fil des ans sont reconstituées ici à partir de documents d'archives. L'ILFI ne parviendra jamais à s'émanciper de la Confédération internationale des syndicats libres (CISL) dont il dépend financièrement. Il a travaillé, pendant 20 ans, à mettre sur pied une cinémathèque, à réaliser différentes productions (dont un seul film), à inciter les syndicats des différents continents à se former à travers les films et à organiser 5 festivals internationaux. Les «moyens audiovisuels » éclipsent peu à peu la suprématie des films. L'article revient sur quelques moments-clés de l'histoire de cet institut et sur quelques films qu'il a promus.

The International Labour Film Institute (ILFI) originated from the international labour movement. It was devoted to workers' education through film. Archived documents reconstitute the conditions of its creation and its activities. The ILFI, which depended financially on the International Confederation of Free Trade Unions (ICFTU), never succeeded in emancipating itself from the confederation. The ILFI functioned for 20 years establishing a film library, producing a variety of visual media (only one film), encouraging trade unions in different continents to use films in union education, and organising 5 international festivals. Little by little, audio-visual means overshadowed the supremacy of film. The article explores key moments of the institute's history and the films it promoted.

\section{INDEX}

Mots-clés : ILFI, CISL, histoire de l'éducation ouvrière, mouvement ouvrier et cinéma

Keywords : ILFI, ICFTU, history of workers' education, labour movement and films

\section{AUTEUR}

\section{FRANÇOISE F. LAOT}

Françoise F. Laot, socio-historienne, est professeure à l'Université de Reims Champagne-Ardenne, membre du Cérep (EA 4692). Elle est également président du Groupe d'étude - Histoire de la formation des adultes (Gehfa).

Ses recherches portent sur l'histoire de l'éducation et de la formation des adultes $\left(\mathrm{XIX}^{\mathrm{e}}-\mathrm{XX}^{\mathrm{e}}\right.$ siècles). Dans ses derniers travaux, elle s'est intéressée à l'analyse sociohistorique de documents filmés et aux revendications internationales pour la formation des travailleuses. Dernier ouvrage : Un film comme source pour l'histoire de la formation des adultes hommes... et femmes. Retour à l'école? (Nancy, 1966), PUN, Éditions universitaires de Lorraine, 2014. 